\title{
TEMPERATURE REGULATED NONLINEAR MICROVALVES FOR SELF-ADAPTIVE MEMS COOLING
}

\author{
Matthew McCarthy ${ }^{1}$, Nicholas Tiliakos ${ }^{3,1}$, Vijay Modi ${ }^{1}$, Luc Fréchette ${ }^{2,1}$ \\ ${ }^{1}$ Columbia University, Department of Mechanical Engineering, New York, NY, 10027, U.S.A. \\ ${ }^{2}$ Université de Sherbrooke, Department of Mechanical Engineering, Sherbrooke, QC, J1K 2R1, Canada \\ ${ }^{3}$ ATK-GASL, Ronkonkoma, NY, 11779, U.S.A.
}

\begin{abstract}
The design and characterization of microfabricated valves for self-adaptive MEMS cooling are presented in this paper. The novel microvalves regulate the flow of a coolant using thermal buckling of eccentric nickel beams, that are solely actuated and controlled by the heat removed, without the need for external wiring or instrumentation. The adaptive microvalves have been modeled, built, and experimentally characterized. The predictions for flow rate as a function of temperature show good agreement with the data while achieving the desired nonlinear valving behavior. Increases in mass flow rate of up to $5 \times 10^{-6} \mathrm{~kg} / \mathrm{s}$ are achieved over temperature ranges of less than $20^{\circ} \mathrm{C}$. These results, as well as the valve fabrication process, can be used to design temperature regulated valve geometries into a distributed micro-cooling scheme.
\end{abstract}

\section{INTRODUCTION}

The current work is focused on developing a thermally adaptive microvalve for flow control in self-regulating MEMS cooling devices. An array of adaptive valves would maintain a surface below some critical temperature by locally modulating flow rate through a cooling scheme in response to changes in heat load. This creates a MEMS skin-cooling scheme similar in nature to its biological inspiration. Figure 1 shows the schematic of a single device as well as an array of devices coating a heated surface.

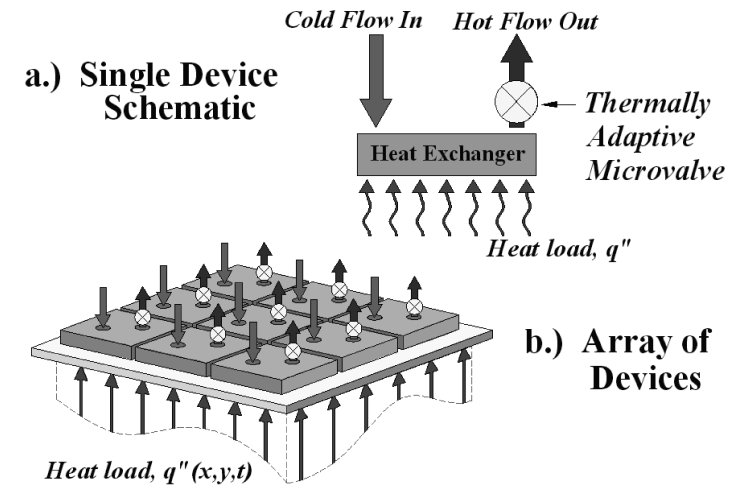

Figure 1. Novel MEMS adaptive cooling scheme. (a) A single device and (c) an array of devices cooling a surface subject to a spatially and temporally varying heat load.

An efficient adaptive microvalve would provide low valve actuation at moderate temperatures and relatively large valve actuation, hence large flow, at high temperatures. A highly nonlinear actuation capability over relatively small temperature ranges would prevent exceeding a critical temperature while minimizing coolant when not required.

The crucial element in this system is a microvalve that adapts to increases in temperature. By using the heat load to drive the thermal actuation, there is no need for control electronics or for resistive heating typically associated with electrothermal actuation [1]. This yields a simple, compact system that can be easily integrated into arrays of devices that need only fluidic connections for coolant.

The mechanism of buckling has been investigated as a means of achieving the nonlinear deflections needed for valve actuation.

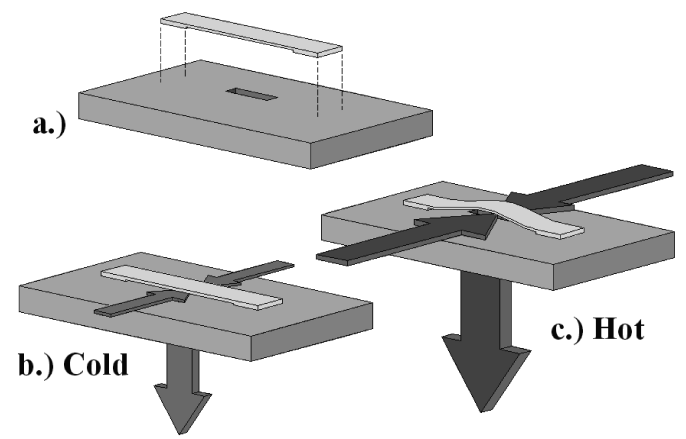

Figure 2. Visualization of thermally adaptive valve concept. (a) Clamped beam fabricated over a slot in substrate with a pressure difference across it (b) at low temperatures, low mass flow through the thin air gap between beam and substrate (c) at high temperatures, the beam buckles, opening the air gap and allowing a large coolant flow rate.

Figure 2 shows conceptually how buckling beam actuation can be used to increase flow rate, where the relative thermal expansion of the beam with respect to the substrate leads to a compressive load in the beam that can induce lateral deflections (buckling). Accordingly, the elastic curve and state of stress of thermally buckled beams was modeled and validated against experimental data in previous work [2]. These findings have been extended in this work to model the flow rate through the contoured beam gap as a function of temperature. This analytic model will be compared to experimental results for multiple valves under various loadings.

\section{THEORY}

The valving mechanism shown in Fig. 2 consists of a thermally buckling beam that increases the thin air gap between itself and the substrate. For small deflections relative to the beam width, the flow through this thin air gap can be modeled as flow through two infinite parallel plates. The valve mass flow rate then varies as the cube of the contoured gap, $d(x)^{3}$, as given by [3]:

$$
\dot{m}=\frac{\Delta P}{6 w v} \int_{a}^{b} d(x)^{3} d x
$$

where $v$ is the kinematic viscosity and $w$ is the parallel plate flow distance underneath the beam. Using Eq. (1), along with the thermal buckling analysis developed in [2], yields

$$
\begin{aligned}
& \frac{\dot{m}}{\Delta P}=\left[\frac{L}{w v}\right]\left(\frac{15 \pi+44}{288 \pi}\right) e^{3}(\sec \eta-1)^{3} \\
& \Delta T=\frac{1}{\alpha}\left(\frac{h}{L}\right)^{2}\left[\frac{\eta^{2}}{3}+\left(\frac{e \pi}{4 h}\right)^{2}(\sec \eta-1)^{2}\right] \quad \eta \equiv \frac{L}{2} \sqrt{P / E I}
\end{aligned}
$$
Hilton Head Island, South Carolina, June 4-8, 2006 
where $\eta$ is the nondimensional axial force, $e$ is the designed beam eccentricity, $\alpha$ is the difference in coefficient of thermal expansion between the beam and substrate and $\Delta T$ is the temperature rise above the zero stress state. The half-length, thickness and moment of inertia of the beam are denoted as $L, h$ and $I$, respectively. Equation (2) shows the mass flow rate per unit driving pressure as a function of axial load, while Eq. (3) gives the beam temperature rise required to generate the nondimensional axial load, $\eta$. As the axial force, $P$, approaches the critical buckling load, $\eta$ approaches $\pi / 2$, and the mass flow rate per unit pressure drop blows up due to the secant term, leading to the desired non-linear valve response.

\section{FABRICATION}

Doubly clamped microfabricated beams suspended over a small air gap were fabricated using a thru mold nickel electroplating process [2]. A shallow silicon etch is previously done to create small 2-3 $\mu \mathrm{m}$ eccentricities in the beam, Fig. 3(b), and a sacrificial photoresist layer is used to form the $1.5 \mu \mathrm{m}$ air gap underneath it. The beam planar dimensions are $4000 \mu \mathrm{m} x$ $700 \mu \mathrm{m}$ with thickness of $25-35 \mu \mathrm{m}$. Deep reactive ion etching from the backside is then used to define the valve slot under the beam, as illustrated in Fig. 2.
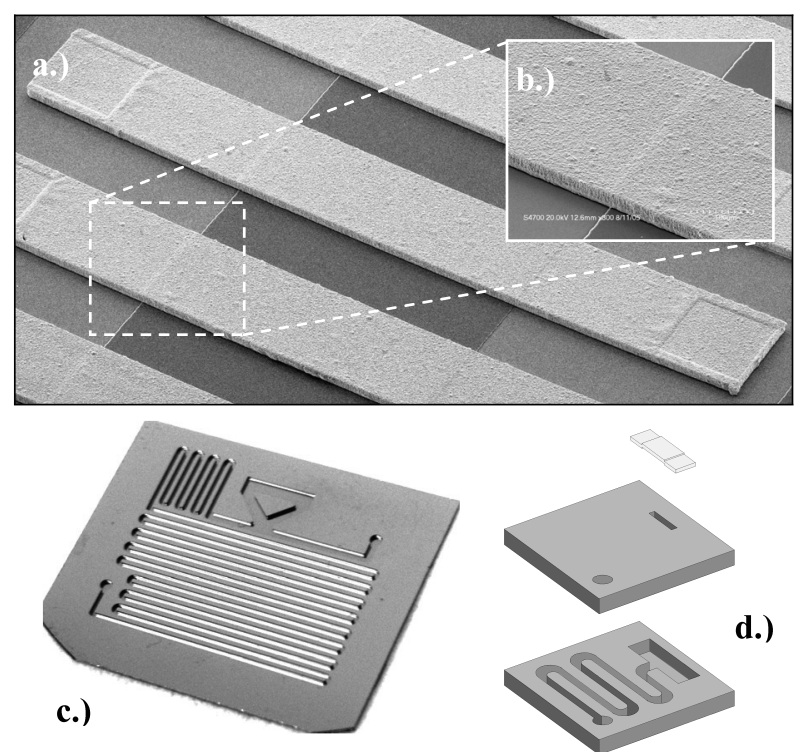

Figure 3. (a) SEM photo of an array of eccentric clamped beams. (b) A beam eccentricity. (c) Deep etched silicon heat exchanger wafer. (d) Assembly of valve wafer and heat exchanger wafer.

The fabricated valve wafer is bonded to another silicon wafer containing a deep reactive ion etched heat exchanger seen in Fig. 3(c). A low temperature photoresist bonding procedure is used due to the temperature sensitive nature of the valves. Figure 3(d) shows the assembly of the two wafers. The valve wafer can be inverted to change the direction of the flow through the valve and of the static pressure acting on the beam.

\section{TESTING \& RESULTS}

The heat exchanger and valve assembly in Fig. 3(d) is used to heat the working fluid (air) to the wall temperature, ensuring that the valve is isothermal. A pressure port, not shown in Fig. 3(d), is fabricated in the device between the heat exchanger and the microvalve. These features allow for the simultaneous control of temperature and pressure drop across the valve during testing. Heat is added to the assembly through a thin film heater while the isothermal temperature is monitored using thermocouples. The pressure drop is maintained constant across the valve while the flow rate is measured. Each valve is tested under positive and negative pressure loadings on the beam.

Eliminating the axial load, $\eta$, Eqs. (2) and (3) are plotted against each other in Fig. 4. This shows the mass flow rate as a function of valve temperature without considering the pressure effects on the beam contour. Experimental data for two valves loaded in both pressure orientations is compared to the model.

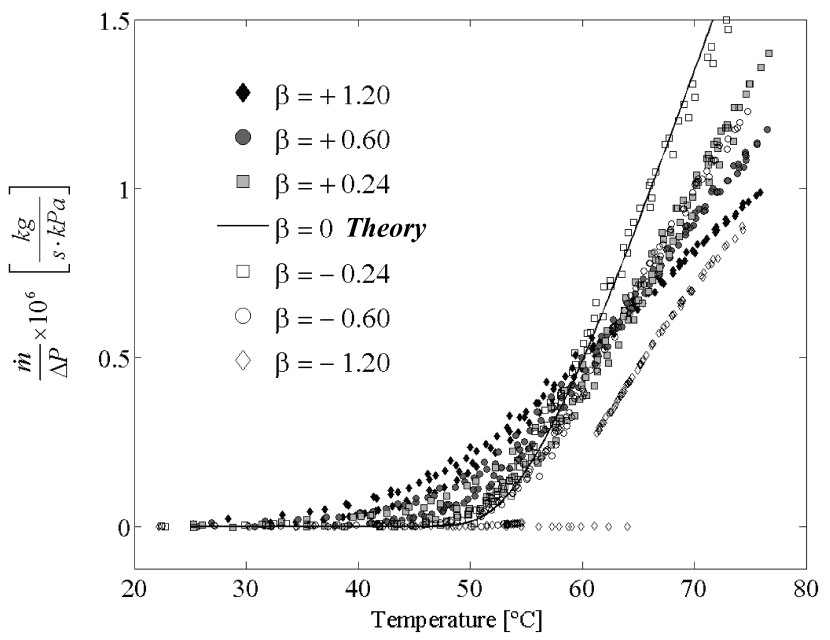

Figure 4. Mass flow rate per unit driving pressure as a function of temperature for various pressure coefficients $\beta=(\Delta P / E)(L / h)^{4}$.

Figure 4 shows that as the magnitude of the pressure coefficient, $\beta$, decreases the experimental results approach the theoretical model for beams buckling with no pressure force. The effect of pressure in the two valve orientations can also be seen. A more complex model is being developed to investigate the effects of pressure loading on the buckling beam contour. The leak rates for the valves tested here were all well below the sensitivity of the mass flow meter used. Calculations based on the valve geometry at ambient conditions suggest leak rates of $\dot{m}_{\text {leak }} / \Delta P \approx 10^{-9} \mathrm{~kg} / \mathrm{s} \cdot \mathrm{kPa}$, three orders of magnitude less than the maximum flow rates achieved.

\section{CONCLUSIONS}

Thermally adaptive microvalves have been modeled, built, and experimentally characterized. Order of magnitude increases in flow rate have been achieved nonlinearly over relatively small temperature ranges, and the effect of pressure can be seen. This nonlinear behavior is the key aspect needed for achieving efficient self-adaptive cooling. These results show that the modeling technique and fabrication process developed herein can be used to build thermally adaptive microvalves with the desired nonlinear actuation capabilities into a MEMS selfregulating cooling scheme.

\section{REFERENCES}

[1] D. Yan, A. Khajepour, and R. Mansour, "Modeling of Two-HotArm Horizontal Thermal Actuator," Journal of Micromechanics and Microengineering, 13, pp. 312-322. (2003)

[2] M. McCarthy, N. Tiliakos, V. Modi, and L. G. Fréchette, "Characterization and Modeling of Thermal Buckling in Eccentrically Loaded Microfabricated Nickel Beams for Adaptive Cooling", Proc. ASME Int'l Mech. Eng. Congress \& Expo., Orlando, FL, Nov. 5-11, 2005, Paper IMECE2005-81415.

[3] R. W. Fox, A. T. McDonald, Introduction to Fluid Mechanics, John Wiley \& Sons. (1998) 